\title{
P-0681- Genetic Association of APOA5 Gene Variant with Diabetic Nephropathy
}

*M.M. Zafar, A.M. Raja, N. Sharif, S. Rahim, M.S. Raja, A. Mahmood, G.K. Raja.

University Institute of Biochemistry \& Biotechnology, Pir Mehr Ali Shah Arid Agriculture University Rawalpindi, 46300, Pakistan

Background: Type 2 Diabetes Mellitus (T2D), characterized by higher circulating glucose levels or insulin insufficiency, is associated with metabolic dysregulations (obesity, hypertension, dyslipidemia etc) with strong environmental and genetic influences (1). T2D is also considered as one of the major risk factor of vascular diseases thus severely compromising the life expectancy. Diabetic nephropathy (DN), a micro vascular complication, affects normal functioning of kidneys in diabetic patients and one of the leading causes of kidney failures (2). Due to high genetic susceptibility of South Asian populations including Pakistanis towards T2D, it could be predicted that they are genetically predisposed to the risk of DN. Apolipoprotein A5 (APOA5) is an important member of gene cluster regulating lipid metabolism. A genetic variant in the promoter region of APOA5 (-1131T>C, rs662799) associates with hyperlipidemia and thus could also contribute to DN (3).

Study Aim: To explore association of rs662799 variant with DN in Pakistani subjects.

Methods: Total 348 individuals of Pakistani ancestry were categorized into three study groups; non-diabetic, non-nephropathic (NDNN), diabetic non-nephropathic (DNN), and diabetic nephropathic (DN). The anthropometric and clinical data were collected. The rs662799 variant (T/C alleles) based genotyping of all study groups was performed through Restriction Fragment Length based Polymerase Chain Reaction (PCR-RFLP) assay using Tru1I (MseI) restriction enzyme. The statistical correlations were explored using SPSS package.

Results \& Discussion: Frequency of the minor $\mathrm{C}$ allele of rs662799 variant did not change much (28-31\%) in three study groups but homozygous $\mathrm{C} / \mathrm{C}$ genotype frequency was slightly raised in DN (25\%) as compared to NDNN and DNN groups (Table 1). No significant association of $\mathrm{C}$ allele found with DN (Table 2). Levels of all clinical variables were raised in DN groups compared to NDNN and DNN (Table 3). The $\mathrm{C}$ allele showed mild yet significant association with elevated triglyceride levels (Table 4).

Table 1: Allele and genotype frequencies of rs662799 variant

\begin{tabular}{lccc}
\hline \multirow{2}{*}{ Alleles } & \multicolumn{3}{c}{$\begin{array}{c}\text { Frequencies (Proportions) } \\
\text { in Study Groups }\end{array}$} \\
\cline { 2 - 4 } & NDNN & DNN & DN \\
\hline $\mathrm{T}$ & 0.72 & 0.69 & 0.70 \\
$\mathrm{C}$ & 0.28 & 0.31 & 0.30 \\
\hline Genotypes & & & \\
\hline $\mathrm{T} / \mathrm{T}$ & 0.61 & 0.60 & 0.65 \\
$\mathrm{~T} / \mathrm{C}$ & 0.22 & 0.17 & 0.10 \\
$\mathrm{C} / \mathrm{C}$ & 0.17 & 0.22 & 0.25 \\
\hline
\end{tabular}

Table 2 : Association of rs662799 variant genotypes with disease status

\begin{tabular}{llcccccc}
\hline & \multicolumn{2}{c}{ NDNN vs DNN } & \multicolumn{2}{c}{ NDNN vs DN } & \multicolumn{2}{c}{ DNN vs DN } \\
\cline { 2 - 8 } Genotypes & OR $(\mathbf{9 5 \%}$ CI $)$ & $\begin{array}{c}\text { p- } \\
\text { valve }\end{array}$ & OR $(\mathbf{9 5 \%}$ CI $)$ & $\begin{array}{c}\text { p- } \\
\text { valve }\end{array}$ & OR (95\% CI) & $\begin{array}{c}\text { p- } \\
\text { valve }\end{array}$ \\
\hline TT vs TC & $0.80(0.36-1.78)$ & 0.61 & $\mathbf{0 . 4 3}(\mathbf{0 . 2 2 - 0 . 8 7 )}$ & $\mathbf{0 . 0 1 6}$ & $0.54(0.22-1.35)$ & 0.4 \\
TT vs CC & $1.30(0.61-2.79)$ & 0.61 & $1.33(0.74-2.39)$ & 0.16 & $1.03(0.48-2.18)$ & 0.4 \\
TT vs TC+CC & $0.78(0.47-1.31)$ & 0.35 & $0.61(0.35-1.05)$ & 0.07 & $1.29(0.70-2.38)$ & 0.6 \\
\hline
\end{tabular}

Table 3: rs662799 variant genotypes based levels (Means \pm SD) of clinical variables in study groups

\begin{tabular}{|c|c|c|c|c|c|c|c|c|c|c|c|c|c|c|c|c|c|c|c|}
\hline \multirow{2}{*}{\multicolumn{2}{|c|}{ Genotypes }} & \multicolumn{6}{|c|}{$\begin{array}{c}\text { NON-DIABETIC NON-NEPHROPATHY } \\
\text { (NDNN) }\end{array}$} & \multicolumn{6}{|c|}{$\begin{array}{c}\text { DIABETIC NON-NEPHROPATHY } \\
\text { (DNN) }\end{array}$} & \multicolumn{6}{|c|}{ DIABETIC NEPHROPATHY (DN) } \\
\hline & & $\begin{array}{c}\text { TG } \\
\text { mg/dl }\end{array}$ & $\begin{array}{l}\mathrm{HDL} \\
\mathrm{mg} / \mathrm{dl}\end{array}$ & $\begin{array}{l}\text { Chol } \\
\text { mg/dl }\end{array}$ & $\begin{array}{c}\mathrm{LDL} \\
\mathrm{mg} / \mathrm{dl}\end{array}$ & UCR & $\begin{array}{l}\text { BUN } \\
\text { mg/dl }\end{array}$ & $\begin{array}{c}\text { TG } \\
\mathrm{mg} / \mathrm{dl}\end{array}$ & $\begin{array}{l}\text { HDL } \\
\mathrm{mg} / \mathrm{dl}\end{array}$ & $\begin{array}{l}\text { Chol } \\
\text { mg/dl }\end{array}$ & $\begin{array}{l}\mathrm{LDL} \\
\mathrm{mg} / \mathrm{dl}\end{array}$ & UCR & $\begin{array}{l}\text { BUN } \\
\mathrm{mg} / \mathrm{dl}\end{array}$ & $\begin{array}{c}\text { TG } \\
\mathrm{mg} / \mathrm{dl}\end{array}$ & $\begin{array}{l}\text { HDL } \\
\mathrm{mg} / \mathrm{dl}\end{array}$ & $\begin{array}{l}\text { Chol } \\
\text { mg/dl }\end{array}$ & $\begin{array}{l}\mathrm{LDL} \\
\mathrm{mg} / \mathrm{dl}\end{array}$ & JCR & $\begin{array}{l}\text { BUN } \\
\mathrm{mg} / \mathrm{dl}\end{array}$ \\
\hline \multirow{2}{*}{$\mathbf{T} / \mathbf{T}$} & ean & 132 & 36.5 & 182.3 & 119.5 & $2 J$ & 19.9 & 191.2 & 37.8 & 172 & 133.8 & 30 & 27.8 & 212.4 & 34.5 & 205 & 156.9 & 1 & 34 \\
\hline & S.D & 30.2 & 6.3 & 31.3 & 25.5 & 15.7 & 6.5 & 56 & 9.5 & 7.7 & 11.8 & 16.1 & 6.5 & 48 & 9.7 & 34.9 & 20.6 & 27.7 & 16.1 \\
\hline \multirow{2}{*}{$\mathbf{C} / \mathbf{T}$} & Mean & 138.8 & 36.6 & 173 & 118.7 & 29.2 & 19.6 & 172.6 & 34.5 & 153 & 127 & 34.2 & 30.2 & 219.6 & 33.9 & 203.9 & 158.6 & 38.8 & 44.2 \\
\hline & S.D & 36.5 & 7.7 & 38.6 & 32.2 & 10.8 & 6 & 24.9 & 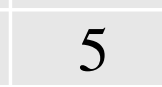 & 19 & 8.9 & 28.1 & 8.5 & 32.5 & 6.5 & 26.4 & 29.3 & 23.3 & 28.1 \\
\hline \multirow{2}{*}{$\mathrm{C} / \mathrm{C}$} & Mean & 137.3 & 37.5 & 187 & 122.1 & 24.3 & 23.3 & 190.6 & 38.1 & 165.5 & 134.7 & 36.4 & 39.3 & 226.8 & 32.8 & 191.7 & 159.7 & 39.2 & 36.4 \\
\hline & S.D & 34.9 & 6.9 & 34.3 & 29.4 & 13.3 & 5.2 & 50.1 & 10 & 32.6 & 8.9 & 26.8 & 5.8 & 28.9 & 5.8 & 21.3 & 30.3 & 25.3 & 26.8 \\
\hline
\end{tabular}

Table 4: Association of rs662799 variant's C allele with risk variables in three study groups

\begin{tabular}{|c|c|c|c|}
\hline \multirow[t]{2}{*}{ Risk Variables } & \multicolumn{3}{|c|}{$\begin{array}{c}\text { Association of risk variables with disease status } \\
\beta \text {-Coefficients }(95 \% \mathrm{CI}), p \text {-values }\end{array}$} \\
\hline & NDNN & DNN & DN \\
\hline \multirow{2}{*}{ Age (Years) } & $1(0.91-$ & $0.98(0.99-1.04)$ & $0.988(0$. \\
\hline & & & \\
\hline \multirow{2}{*}{ BMI $\left(\mathbf{K g} / \mathbf{m}^{2}\right)$} & $1.06(0.46-2.44)$ & $0.93(0.335-2.5)$ & $2.5(0.76-7.8)$ \\
\hline & 0.081 & 0.088 & 0.017 \\
\hline \multirow{2}{*}{$\begin{array}{c}\text { Dyslipidemia } \\
\text { (mg/dL) }\end{array}$} & $0.98(0.929-1.04)$ & $0.93(0.85-1.01)$ & $1.1(0.96-1.25)$ \\
\hline & & & \\
\hline \multirow{2}{*}{$\begin{array}{l}\text { Triglycerides } \\
\text { (mg/dL) }\end{array}$} & $0.972(0.88-1.06)$ & $1.03(0.99-1.00)$ & $1.82(1.59-2.06)$ \\
\hline & 0.036 & 0.029 & 0.043 \\
\hline \multirow{2}{*}{$\begin{array}{l}\text { Hypertension } \\
\text { (mmHg) }\end{array}$} & $0.84(0.39-1.83)$ & $1.25(0.51-3.27)$ & $0.596(0.220-1.16)$ \\
\hline & 0.675 & 0.584 & 0.309 \\
\hline \multirow{2}{*}{$\begin{array}{c}\text { Urea:Creatinine } \\
\text { Ratio }\end{array}$} & $0.98(0.891 .07)$ & $\begin{array}{l}0.933(0.85- \\
1.018)\end{array}$ & $1.007(0.96-1.054)$ \\
\hline & 0.06 & 0.019 & 0.07 \\
\hline
\end{tabular}

This work was supported by the research grant f20-2255/NRPU/R\&D/HEC by

Higher Education Commission of Pakistan
Conclusions: The minor $\mathrm{C}$ allele of rs662799 variant lacked direct association with DN in Pakistani subjects. Though in heterozygous status, TC genotype, it seemed to show a protective effect from disease. However the $\mathrm{C}$ allele seemed to increase the risk of triglyceridemia in an additive manner. Our results are in accordance with previous studies on other world populations.

References:1.Keramat L, Sadrzadeh-Yeganeh H, Sotoudeh G, Zamani E, Eshraghian M, Mansoori A, et al. Apolipoprotein A2- $265 \mathrm{~T}>\mathrm{C}$ polymorphism interacts with dietary fatty acids intake to modulate inflammation in type 2 diabetes mellitus patients. Nutrition. 2017;37:86-91.

2.Perreault L, Pan Q, Aroda V, Barrett-Connor E, Dabelea D, Dagogo-Jack S, et al. Exploring residual risk for diabetes and microvascular disease in the Diabetes Prevention Program Outcomes Study (DPPOS). Diabetic Medicine. 2017.

3.Mahrooz A, Zargari M, Ansari V, Makhlough A, Hashemi-Sooteh M-B. Association of APOA5 Gene Promoter Region-1131T> C Polymorphism (rs662799) to Plasma Triglyceride Level in Patients with Type 2 Diabetic Nephropathy. Journal of clinical and diagnostic research: JCDR. 2016;10(5):BC09. 